\title{
Physical Properties of Polychromatic Quasi Crystals
}

\author{
M. Radha Madhavi \\ Sr. Associate Professor \\ Department of Mathematics \\ Aarupadai Veedu Institute of Technology \\ Vinayaka Missions University \\ Chennai, INDIA \\ mrmadhavi5@gmail.com
}

\author{
D.Bharathi \\ Professor \\ Department of Engg. Mathematics \\ A.U.College of Engineering \\ Andhra University \\ Visakhapatnam, INDIA \\ bharathidora57@yahoo.co.in
}

\begin{abstract}
The polychromatic point groups with the help of crystallographic point groups containing one dimensional complex irreducible representations of generator groups were derived by Indenbom et al (1960). Rama Mohana Rao K (1987) had studied the magnetic properties pertaining to the ten crystallographic point groups that generate the 18 polychromatic point groups has already been covered during the study of the 90 magnetic classes. In this paper 18 polychromatic point groups with the help of quasi crystals with $5,8,10$ \& 12 symmetries are derived. The 54 polychromatic classes with the help of 11 non-crystallographic point groups containing one dimensional complex irreducible representations are given. The maximum number of nonvanishing independent constants required to describe a chosen physical property by the 54 polychromatic classes by using Bhagavantham's formula are determined and tabulated these constants to 10 known physical properties.
\end{abstract}

Keywords: Quasi Crystals, Polychromatic point group, Magnetic properties, Irreducible representation

\section{INTRODUCTION}

The discovery of icosahedral quasi crystals in Al-Mn alloys by Schechtman et al (1984), other quasi crystals with ten, twelve, eight and five-fold symmetries has been obtained in succession. Levine and Steinhart (1984) introduced the term quasi crystals for these unusual systems exhibiting diffraction patterns with non-crystallographic symmetry. The decagonal symmetry found in $\mathrm{NiCr}$, the octagonal symmetry in NiSi and the pentagonal symmetry found in AlCoNiTb. Feng and co-workers have discovered an incommensurate structure with cubic point group symmetry in V-Ni-Si alloys. Janssen gave a clear theoretical explanation for quasi periodic structures which may have either crystallographic or non-crystallographic point group symmetry. They can be obtained by projecting a higher dimensional periodic structure upon the physical space or by cutting a higher dimensional structure. Bradnd muller \& Clauss (1988) Provided the irreducible tensors of rank 1-4 for all the irreducible representations of the pentagonal and icosahedral point groups. He also gave the Raman and hyper - Raman tensors for groups with five-fold symmetry.

Qusai crystals possess various point group symmetries. So many researchers worked on the physical properties of quasi crystals in both theoretical and experimental aspects. Some researchers derived the physical properties of quasi crystals using group theoretical methods. In chapter 2 we gave the number of independent constants required to describe a physical property of quasi crystals with $5,7,8,10,12$ and icosahedral symmetries and also the number of independent constants required to describe a physical property of ferroic species of quasi crystals with 5,7,8,10,12 and icosahedral symmetries using Bhagavantham's formula.

Belov and Tarkhova (1956) gave the interpretation of anti-symmetry as two colour symmetry and also gave the concept of polychromatic symmetry. Consequently in 1960 Indenbom et al derived the 18 poly chromatic point groups by introducing the colour change operation $R_{n}, n=3,4$ or 6 with $R_{n}{ }^{n}=E$ with the help of 10 crystallographic point groups containing one dimensional complex irreducible representations and associated with the 18 pairs of one dimensional complex irreducible representations of the generator groups. 


\section{Radha Madhavi \& D.Bharathi}

Naish (1963) described that the polychromatic structure, and hence the polychromatic point groups and space groups have a useful role to play in many physical applications such as the derivation and description of similarity symmetry point groups and space groups, in description of stem and layer symmetry groups in higher dimensional space and describing the magnetic symmetry and screw structure and where the traditional magnetic groups cannot adequately describe the situation.

Rama Mohana Rao K (1987) had studied the magnetic properties pertaining to the ten crystallographic point groups that generate the 18 polychromatic point groups has already been covered during the study of the 90 magnetic classes. He studied the 18 polychromatic point groups whose geometric configuration was provided by Indenbom et al and Bradley and Cracknel with a view to enumerating the number of constants required to describe a chosen magnetic property and occurring before an irreducible representation of the variant by G. In 1988 the same author obtained the maximum number of non-vanishing independent constants required to describe a chosen physical property by the 18 polychromatic classes and he tabulated the constants of six known physical properties. He also studied theoretically as the effect of spins in crystals in their magnetic state on the non-magnetic physical properties.

In this paper the concept of 18 polychromatic point groups with the help of 10 crystallographic point groups containing one dimensional complex irreducible representations (Indenbom et al) was extended to the quasi crystals with 5,8,10 \& 12 symmetries. The 54 polychromatic classes with the help of 11 non-crystallographic point groups containing one dimensional complex irreducible representations are given. The maximum number of non-vanishing independent constants required to describe a chosen physical property by the 54 polychromatic classes by using Bhagavantham's formula are determined and tabulated these constants to 10 known physical properties.

\section{Polychromatic Classes of Quasi Crystals}

The polychromatic point group $\mathrm{C}$, is given by

$$
C=\alpha_{1} H+\alpha_{2} R_{p} H+\alpha_{3} R_{p}^{2} H+------+\alpha_{p} R_{p}^{p-1} H
$$

Where $\mathrm{H}$ is a subgroup of some point group $\mathrm{G}$ and is of order $(1 / \mathrm{p}) \times$ the order of $\mathrm{G}$ and $\alpha_{1}, \alpha_{2}, \alpha_{3}-$ ,$--- \alpha_{p}$ are the left coset representatives in the expansion of $\mathrm{G}$.

$$
G=\alpha_{1} H+\alpha_{2} H+\alpha_{3} H+------+\alpha_{p} H
$$

If $\mathrm{p}=3,4$ or 6 we can derive p-coloured point groups. Bradley and cracknel gave the 58 black and white point groups are derived by studying the real one dimensional representation of the ordinary point groups and multiplying an element whose character is -1 by the colour changing operation. In the same way it is possible to use the complex one dimensional representation of the ordinary point groups to derive the 3-coloured,4-coloured and 6-coloured point groups(Indenbom et al). The complex numbers $\omega\left(=e^{\frac{2 \pi i}{3}}\right), i\left(=e^{\frac{2 \pi i}{4}}\right)$ and $-\omega^{*}\left(=-e^{\frac{2 \pi i}{6}}\right)$ can be associated with colour changing operations for 3-coloured,4-coloured and 6-coloured point groups respectively. The two members of a pair of complex conjugate representations of an ordinary point group both lead to the same polychromatic point group.

Similarly we defined 5-coloured,8-coloured 10-coloured and 12-coloured point groups by taking the complex one dimensional representation of the quasi crystals. The complex numbers

$\rho\left(=e^{\frac{2 \pi i}{5}}\right), \rho\left(=e^{\frac{2 \pi i}{8}}\right), \rho\left(=e^{\frac{2 \pi i}{10}}\right), \rho\left(=e^{\frac{2 \pi i}{12}}\right)$, can associated with colour changing operations for 5-coloured,8coloured 10-coloured and 12-coloured point groups respectively. All the 54 polychromatic classes of quasi crystals are given in table 1.

Table 1. 54 polychromatic variants of quasi crystals

\begin{tabular}{|l|l|l|l|l|l|l|l|l|}
\hline S.NO & $\begin{array}{l}\mathrm{C} \text { (poly } \\
\text { chromatic } \\
\text { point group) }\end{array}$ & $\begin{array}{l}\mathrm{G} \text { (point } \\
\text { group) }\end{array}$ & S.NO & $\begin{array}{l}\mathrm{C} \text { (poly } \\
\text { chromatic } \\
\text { point group) }\end{array}$ & $\begin{array}{l}\mathrm{G} \\
\text { (point } \\
\text { group) }\end{array}$ & $\begin{array}{l}\text { S.NO } \\
\begin{array}{l}\mathrm{C}(\text { poly } \\
\text { chromatic } \\
\text { point group) }\end{array}\end{array}$ & $\begin{array}{l}\text { G } \\
\text { (point } \\
\text { group) }\end{array}$ \\
\hline 1 & $5_{1}^{(5)}$ & 5 & 19 & $10 / m^{(5)}$ & $10 / \mathrm{m}$ & 37 & $12_{3}^{(12)}$ & 12 \\
\hline 2 & $5_{2}^{(5)}$ & 5 & 20 & $10 / m^{(5)}$ & $10 / \mathrm{m}$ & 38 & $12_{1}^{(6)}$ & 12 \\
\hline
\end{tabular}


Physical Properties of Polychromatic Quasi Crystals

\begin{tabular}{|l|c|c|l|c|l|l|l|l|}
\hline \hline 3 & $\overline{5}_{1}^{(5)}$ & $\overline{5}$ & 21 & $10 / m^{\prime \prime}(5)$ & $10 / \mathrm{m}$ & 39 & $12_{2}^{(6)}$ & 12 \\
\hline 4 & $\overline{5}_{2}^{(5)}$ & $\overline{5}$ & 22 & $10 / m^{\prime \prime(5)}$ & $10 / \mathrm{m}$ & 40 & $\overline{12}_{1}^{(12)}$ & $\overline{12}$ \\
\hline 5 & $\overline{5}_{1^{\prime}}^{(5)}$ & $\overline{5}$ & 23 & $8_{1}^{(8)}$ & 8 & 41 & $\overline{12}_{2}^{(12)}$ & $\overline{12}$ \\
\hline 6 & $\overline{5}_{2^{\prime}}^{(5)}$ & $\overline{5}$ & 24 & $8_{2}^{(8)}$ & 8 & 42 & $\overline{12}_{3}^{(12)}$ & $\overline{12}$ \\
\hline 7 & $10_{1}^{(10)}$ & 10 & 25 & $8_{1}^{(4)}$ & 8 & 43 & $\overline{12}_{1}^{(6)}$ & $\overline{12}$ \\
\hline 8 & $10_{2}^{(10)}$ & 10 & 26 & $\overline{8}_{1}^{(8)}$ & $\overline{8}$ & 44 & $\overline{12}_{2}^{(6)}$ & $\overline{12}$ \\
\hline 9 & $10_{1}^{(5)}$ & 10 & 27 & $\overline{8}_{2}^{(8)}$ & $\overline{8}$ & 45 & $12 / m^{(12)}$ & $12 / \mathrm{m}$ \\
\hline 10 & $10_{1}^{(5)}$ & 10 & 28 & $\overline{8}_{1}^{(4)}$ & $\overline{8}$ & 46 & $12 / m^{\prime(12)}$ & $12 / \mathrm{m}$ \\
\hline 11 & $\overline{10}_{1}^{(10)}$ & $\overline{10}$ & 29 & $8 / m^{(8)}$ & $8 / \mathrm{m}$ & 47 & $12 / m^{\prime \prime(12)}$ & $12 / \mathrm{m}$ \\
\hline 12 & $\overline{10}_{2}^{(10)}$ & $\overline{10}$ & 30 & $8 / m^{\prime(8)}$ & $8 / \mathrm{m}$ & 48 & $12 / m^{\prime \prime \prime(12)}$ & $12 / \mathrm{m}$ \\
\hline 13 & $\overline{10}_{1}^{(5)}$ & $\overline{10}$ & 31 & $8 / m^{\prime(8)}$ & $8 / \mathrm{m}$ & 49 & $12 / m^{1 V^{(12)}}$ & $12 / \mathrm{m}$ \\
\hline 14 & $\overline{10}_{2}^{(5)}$ & $\overline{10}$ & 32 & $8 / m^{(4)}$ & $8 / \mathrm{m}$ & 50 & $12 / m^{(6)}$ & $12 / \mathrm{m}$ \\
\hline 15 & $10 / m^{(10)}$ & $10 / \mathrm{m}$ & 33 & $8 / m^{\prime(4)}$ & $8 / \mathrm{m}$ & 51 & $12 / m^{\prime(6)}$ & $12 / \mathrm{m}$ \\
\hline 16 & $10 / m^{\prime(10)}$ & $10 / \mathrm{m}$ & 34 & $8 / m^{\prime(4)}$ & $8 / \mathrm{m}$ & 52 & $12 / m^{\prime(6)}$ & $12 / \mathrm{m}$ \\
\hline 17 & $10 / m^{\prime(10)}$ & $10 / \mathrm{m}$ & 35 & $12_{1}^{(12)}$ & 12 & 53 & $12 / m^{\prime \prime(6)}$ & $12 / \mathrm{m}$ \\
\hline 18 & $10 / m^{\prime \prime \prime(10)}$ & $10 / \mathrm{m}$ & 36 & $12_{2}^{(12)}$ & 12 & 54 & $12 / m^{1 V^{(6)}}$ & $12 / \mathrm{m}$ \\
\hline
\end{tabular}

\section{Physical Properties of Quasi Crystals having Polychromatic Symmetry}

The physical property of substances depends on the relation between two quantities such as scalarscalar, scalar-vector or symmetry tensor of rank two etc. In all the cases, each of the relation requires a certain number of independent constants connecting the components of the quantities involved for its complete description. The number of independent constants required to describe a physical property depends on the property chosen and on the crystallography symmetry of the material in solid state.

Bhagavantam [4] and other researchers gave the number of independent constants to describe the physical properties of the crystallographic point groups by using formula

$$
n_{i}=\frac{1}{N} \sum_{j} h_{j} \chi_{j}^{\prime}(R) \chi_{i}(R)
$$

Where $\mathrm{N}$ is the total number of elements of the group $\mathrm{G}$ and $\mathrm{h}_{\mathrm{j}}$ is the number of elements in the $\mathrm{j}$ th class. $\chi_{j}^{\prime}(R)$ is the character of the appropriate transformation matrix relative to the operation $R$ and $\chi_{i}$ $(\mathrm{R})$ is the character relative to the operation $\mathrm{R}$ in the particular irreducible representation of a group $\mathrm{G}$. The classifications of ten physical properties are listed in table 3 as given by Bhagavantam (1966).

For example, the thermal conductivity or electrical resistivity $\chi_{\rho}(R)$ is given by

$\chi_{\rho}(\mathrm{R})=4 \cos ^{2} \phi \pm 2 \cos \phi$

The maximum number of independent constants to describe the thermal conductivity is 6 . Consider the point group 5, which contains two pairs of one dimensional complex representation.

Table2. The character table for a point group 5

\begin{tabular}{|c|l|l|l|l|l|l|}
\hline $5\left(\mathrm{C}_{5}\right)$ & \multicolumn{1}{|c|}{$\mathrm{E}$} & $\mathrm{C}_{5}$ & \multicolumn{1}{c|}{$C_{5}^{2}$} & \multicolumn{1}{c|}{$C_{5}^{3}$} & $C_{5}^{4}$ & $\mathrm{n}_{\mathrm{i}}$ \\
\hline $\mathrm{A}$ & 1 & 1 & 1 & 1 & 1 & 2 \\
\hline \multirow{2}{*}{$\mathrm{E}_{1}$} & 1 & $\rho$ & $\rho^{2}$ & $\rho^{-2}$ & $\rho^{-1}$ & 1 \\
\cline { 2 - 8 } & 1 & $\rho^{-1}$ & $\rho^{-2}$ & $\rho^{2}$ & $\rho$ & 1 \\
\hline \multirow{2}{*}{$\mathrm{E}_{2}$} & 1 & $\rho^{2}$ & $\rho^{-1}$ & $\rho$ & $\rho^{-2}$ & 1 \\
\cline { 2 - 8 } & 1 & $\rho^{-2}$ & $\rho$ & $\rho^{-1}$ & $\rho^{2}$ & 1 \\
\hline$\chi_{\rho}(\mathrm{R})$ & 6 & 1 & 1 & 1 & 1 & \\
\hline
\end{tabular}




\section{Radha Madhavi \& D.Bharathi}

The polychromatic variants of the point group 5 are $5^{(5)}$ and $5^{\text {,(5) }}$,by using eq. (1) the independent thermal conductivity constants of $5^{(5)}$ and $5^{,(5)}$ is one. Similarly the number of independent constants to describe the physical properties of all 54 polychromatic variants are tabulated in table 4 .

Table 3. Classification of physical properties

\begin{tabular}{|c|c|c|c|c|}
\hline S.NO & Physical property & Character $\chi_{j}^{\prime}(\mathbf{R})$ & $\chi_{\mathrm{i}}(\mathbf{R})$ & Max.No.of constants \\
\hline 1 & $\begin{array}{l}\text { Pyro electricity: production } \\
\text { of charges by hydrostatic } \\
\text { pressure }\end{array}$ & $2 \cos \phi \pm 1$ & 1 & 3 \\
\hline 2 & $\begin{array}{l}\text { Electrical resistivity, } \\
\text { thermal conductivity }\end{array}$ & $4 \cos ^{2} \phi \pm 2 \cos \phi$ & 1 & 6 \\
\hline 3 & Hall Effect & $4 \cos ^{2} \phi \pm 4 \cos \phi+1$ & 1 & 9 \\
\hline 4 & Piezoelectricity & $\left(4 \cos ^{2} \phi \pm 2 \cos \phi\right)(2 \cos \phi \pm 1)$ & 1 & 18 \\
\hline 5 & Photoelasticity & $16 \cos ^{4} \phi \pm 16 \cos ^{3} \phi+4 \cos ^{2} \phi$ & 1 & 36 \\
\hline 6 & $\begin{array}{l}\text { Elasticity(Symmetric } \\
\& \text { symmetric tensor) }\end{array}$ & $16 \cos ^{4} \phi \pm 8 \cos ^{3} \phi-4 \cos ^{2} \phi+1$ & 1 & 21 \\
\hline 7 & $\begin{array}{l}\text { Magnetothermoelectric } \\
\text { power, piezo-Hall effect }\end{array}$ & $16 \cos ^{4} \phi \pm 24 \cos ^{3} \phi+12 \cos ^{2} \phi \pm 2 \cos \phi$ & 1 & 54 \\
\hline 8 & $\begin{array}{l}\text { Piezoelectric } \\
\text { Coefficients } \\
\text { (Higher order ) }\end{array}$ & $32 \cos ^{5} \phi \pm 32 \cos ^{4} \phi \mp 4 \cos ^{2} \phi+2 \cos \phi \pm 1$ & 1 & 63 \\
\hline 9 & $\begin{array}{l}\text { Elastic } \\
\text { Coefficients } \\
\text { (Higher order ) }\end{array}$ & $\begin{array}{l}64 \cos ^{6} \phi \pm 32 \cos ^{5} \phi-48 \cos ^{4} \phi \\
\mp 8 \cos ^{3} \phi+16 \cos ^{2} \phi\end{array}$ & 1 & 56 \\
\hline 10 & $\begin{array}{l}\text { Photo elastic } \\
\text { Coefficients } \\
\text { (Higher order) }\end{array}$ & $\begin{array}{l}64 \cos ^{6} \phi \pm 64 \cos ^{5} \phi \\
\mp 8 \cos ^{3} \phi+4 \cos ^{2} \phi \pm 2 \cos \phi\end{array}$ & 1 & 126 \\
\hline
\end{tabular}

Table 4. Number of independent constants to describe the physical properties by the 54 polychromatic classes of quasi crystals

\begin{tabular}{|c|c|c|c|c|c|c|c|c|c|c|c|}
\hline \multirow[t]{2}{*}{ S.NO } & \multirow{2}{*}{$\begin{array}{l}\text { Polychromatic } \\
\text { class }\end{array}$} & \multicolumn{10}{|c|}{ Number of independent constants to describe the physical properties in table 7.2} \\
\hline & & 1 & 2 & 3 & 4 & 5 & 6 & 7 & 8 & 9 & 10 \\
\hline 1 & $5_{1}^{(5)}$ & 1 & 1 & 2 & 4 & 7 & 4 & 11 & 13 & 11 & 25 \\
\hline 2 & $5_{?}^{(5)}$ & 0 & 1 & 1 & 3 & 7 & 4 & 10 & 12 & 11 & 25 \\
\hline 3 & $\overline{5}_{1}^{(5)}$ & 0 & 1 & 2 & 0 & 7 & 4 & 11 & 0 & 11 & 25 \\
\hline 4 & $\overline{5}_{2}^{(5)}$ & 0 & 1 & 1 & 0 & 7 & 4 & 10 & 0 & 11 & 25 \\
\hline 5 & $\overline{5}_{1}^{(5)}$ & 1 & 0 & 0 & 4 & 0 & 0 & 0 & 13 & 0 & 0 \\
\hline 6 & $\overline{5^{\prime}}$ & 0 & 0 & 0 & 3 & 0 & 0 & 0 & 12 & 0 & 0 \\
\hline 7 & $10_{1}^{(10)}$ & 1 & 1 & 2 & 4 & 6 & 3 & 10 & 11 & 7 & 18 \\
\hline 8 & $10_{2}^{(10)}$ & 0 & 1 & 1 & 2 & 5 & 3 & 7 & 7 & 7 & 16 \\
\hline 9 & $10_{1}^{(5)}$ & 0 & 0 & 0 & 1 & 2 & 1 & 3 & 5 & 4 & 9 \\
\hline 10 & $10_{1}^{(5)}$ & 0 & 0 & 0 & 0 & 1 & 1 & 1 & 2 & 4 & 7 \\
\hline 11 & $\overline{10}_{1}^{(10)}$ & 1 & 0 & 0 & 4 & 1 & 1 & 1 & 11 & 4 & 7 \\
\hline 12 & $\overline{10}_{2}^{1}$ & 0 & 1 & 1 & 1 & 5 & 3 & 7 & 5 & 7 & 16 \\
\hline 13 & $\frac{2}{10_{1}^{(5)}}$ & 0 & 1 & 2 & 0 & 6 & 3 & 10 & 2 & 7 & 18 \\
\hline 14 & $\overline{10_{2}^{(5)}}$ & 0 & 0 & 0 & 0 & 2 & 1 & 3 & 7 & 4 & 9 \\
\hline 15 & $10 / m^{(10)}$ & 0 & 1 & 2 & 0 & 6 & 3 & 10 & 2 & 7 & 18 \\
\hline 16 & $10 / m^{\prime(10)}$ & 0 & 1 & 1 & 0 & 5 & 3 & 7 & 0 & 7 & 17 \\
\hline 17 & $10 / m^{\prime \prime(10)}$ & 0 & 0 & 0 & 0 & 2 & 1 & 3 & 0 & 4 & 9 \\
\hline 18 & $10 / m^{\prime \prime \prime(10) ~}$ & 0 & 0 & 0 & 0 & 1 & 1 & 1 & 0 & 4 & 8 \\
\hline 19 & $10 / m^{(5)}$ & 1 & 0 & 0 & 4 & 0 & 0 & 0 & 11 & 0 & 0 \\
\hline 20 & $10 / m^{\prime(5)}$ & 0 & 0 & 0 & 2 & 0 & 0 & 0 & 7 & 0 & 0 \\
\hline 21 & $10 / m^{\prime(5)}$ & 1 & 0 & 0 & 1 & 0 & 0 & 0 & 5 & 0 & 0 \\
\hline 22 & $10 / m^{\prime \prime(5)}$ & 0 & 0 & 0 & 0 & 0 & 0 & 0 & 2 & 0 & 0 \\
\hline 23 & $8_{1}^{(8)}$ & 1 & 1 & 2 & 4 & 6 & 3 & 10 & 10 & 6 & 16 \\
\hline
\end{tabular}


Physical Properties of Polychromatic Quasi Crystals

\begin{tabular}{|c|c|c|c|c|c|c|c|c|c|c|c|}
\hline 24 & $8_{2}^{(8)}$ & 0 & 1 & 1 & 2 & 5 & 3 & 7 & 7 & 8 & 17 \\
\hline 25 & $8_{1}^{(4)}$ & 0 & 0 & 0 & 1 & 2 & 1 & 3 & 7 & 6 & 13 \\
\hline 26 & $\overline{8}_{1}^{(8)}$ & 1 & 0 & 0 & 1 & 2 & 1 & 3 & 7 & 6 & 13 \\
\hline 27 & $\overline{8}_{2}^{(8)}$ & 0 & 1 & 1 & 2 & 5 & 3 & 7 & 7 & 8 & 17 \\
\hline 28 & $\overline{8}_{1}^{(4)}$ & 0 & 1 & 2 & 4 & 6 & 3 & 10 & 10 & 6 & 16 \\
\hline 29 & $8 / m^{(8)}$ & 0 & 1 & 2 & 0 & 6 & 3 & 10 & 0 & 6 & 16 \\
\hline 30 & $8 / m^{\prime(8)}$ & 0 & 1 & 1 & 0 & 5 & 3 & 7 & 0 & 8 & 17 \\
\hline 31 & $8 / m^{\prime \prime(8)}$ & 0 & 0 & 0 & 0 & 2 & 1 & 3 & 0 & 6 & 13 \\
\hline 32 & $8 / m^{(4)}$ & 1 & 0 & 0 & 4 & 0 & 0 & 0 & 10 & 0 & 0 \\
\hline 33 & $8 / m^{\prime(4)}$ & 0 & 0 & 0 & 2 & 0 & 0 & 0 & 7 & 0 & 0 \\
\hline 34 & $8 / m^{\prime \prime(4)}$ & 0 & 0 & 0 & 1 & 0 & 0 & 0 & 7 & 0 & 0 \\
\hline 35 & $12_{1}^{(12)}$ & 1 & 1 & 2 & 4 & 6 & 3 & 10 & 11 & 7 & 18 \\
\hline 36 & $12_{2}^{(12)}$ & 0 & 1 & 1 & 2 & 5 & 3 & 7 & 7 & 7 & 16 \\
\hline 37 & $12_{3}^{(12)}$ & 0 & 0 & 0 & 1 & 2 & 1 & 3 & 5 & 4 & 9 \\
\hline 38 & $12_{1}^{(6)}$ & 0 & 0 & 0 & 0 & 1 & 1 & 1 & 2 & 3 & 6 \\
\hline 39 & $12_{2}^{(6)}$ & 0 & 0 & 0 & 0 & 0 & 0 & 0 & 1 & 1 & 2 \\
\hline 40 & $\overline{12}_{1}^{(12)}$ & 1 & 0 & 0 & 4 & 0 & 0 & 0 & 11 & 1 & 2 \\
\hline 41 & $\overline{12}_{2}^{(12)}$ & 0 & 1 & 1 & 0 & 5 & 3 & 7 & 2 & 7 & 16 \\
\hline 42 & $\overline{12}_{3}^{(12)}$ & 0 & 0 & 0 & 1 & 2 & 1 & 3 & 5 & 4 & 9 \\
\hline 43 & $\overline{12}_{1}^{(6)}$ & 0 & 0 & 0 & 2 & 1 & 1 & 1 & 7 & 3 & 6 \\
\hline 44 & $\overline{12}_{2}^{(6)}$ & 0 & 1 & 2 & 0 & 6 & 3 & 10 & 1 & 7 & 18 \\
\hline 45 & $12 / m^{(12)}$ & 0 & 1 & 2 & 0 & 6 & 3 & 10 & 0 & 7 & 18 \\
\hline 46 & $12 / m^{\prime(12)}$ & 0 & 1 & 1 & 0 & 5 & 3 & 7 & 0 & 7 & 16 \\
\hline 47 & $12 / m^{\prime \prime(12)}$ & 0 & 0 & 0 & 0 & 2 & 1 & 3 & 0 & 4 & 9 \\
\hline 48 & $12 / \mathrm{m}^{\prime \prime(12)}$ & 0 & 0 & 0 & 0 & 1 & 1 & 1 & 0 & 3 & 6 \\
\hline 49 & $12 / m^{1 V^{(12)}}$ & 0 & 0 & 1 & 0 & 0 & 0 & 0 & 0 & 1 & 2 \\
\hline 50 & $12 / m^{(6)}$ & 1 & 0 & 0 & 4 & 0 & 0 & 0 & 11 & 0 & 0 \\
\hline 51 & $12 / \mathrm{m}^{\prime(6)}$ & 0 & 0 & 0 & 2 & 0 & 0 & 0 & 7 & 0 & 0 \\
\hline 52 & $12 / \mathrm{m}^{(6)}$ & 0 & 0 & 0 & 1 & 0 & 0 & 0 & 5 & 0 & 0 \\
\hline 53 & $12 / m^{\prime \prime \prime(6)}$ & 0 & 0 & 0 & 0 & 0 & 0 & 0 & 2 & 0 & 0 \\
\hline 54 & $12 / m^{1 V^{(6)}}$ & 0 & 0 & 0 & 0 & 0 & 0 & 0 & 1 & 0 & 0 \\
\hline
\end{tabular}

\section{Conclusion}

In this paper the theoretical study of non vanishing independent constants of physical properties of quasi crystals with polychromatic symmetry are discussed. The 54 polychromatic classes with help of 11 non-crystallographic point groups are tabulated and Using Bhagavantam's formula, the number of independent constants needed to describe a physical property of quasi crystals with polychromatic symmetry are calculated. The experimental investigations suggests to a physicist to identify the quasi crystals possessing the symmetry of the polychromatic groups.

\section{REFERENCES}

[1] Belov N V and Tarhova T N, Kristallogr.14, 1956

[2] Indenbom V L, Kristallogr. 5 ,513, 1960

[3] Indenbom V L, Belov N V and Neronova N N , Kristallogr. 5, 497, 1960

[4] Naish V E , Izv.Akad.Nauk 27 1496, 1963 (Bull.Acad.Sci.USSR.Phys.Ser.27 1468)

[5] Rama Mohana Rao K, J.Phys A: Maths.Gen 18 739,1985.

[6] Rama Mohana Rao K, J.Phys A: Maths.Gen 20 47-57,1987.

[7] Rama Mohana Rao K ,J.Phys A: Maths.Gen 21 1513-1518, 1988

[8] Leviene D and Steinhardt P J Phy.Rev.Lett 53 2477-80, 1984

[9] Penrose R , Bull.Inst.Math.Appl. 10 166-171, 1974 
[10] Penrose R , Maths.Intelligener 2 32-37, 1979

[11] de Bruijn N G , Proc.Konig.ned.Akad.Weten. A 84 39-66,1981

[12] Feng. C, G.Lu and R.L.withers 1989 J.Phys: condens. Matter 13695.

[13] Feng. C, G.Lu H.Q.Ye, K.H.Kuo, R.L.withers and G.Van Fend eloo, J.Phys: condens. Matter 2 9749, 1990

[14] Brandmuller J and claus R , Ind.Pure.Appl.Phy 26,60,1988

[15] Scechman.D, Blech.I, Gratias.D and Cahn J W, Phy.Rev.Lett.53, 1951-53, 1984.

[16] Janssen T, Z.Kristallogra.198,17- 32, 1992.

\section{AUTHOR's BIOGRAPHY}

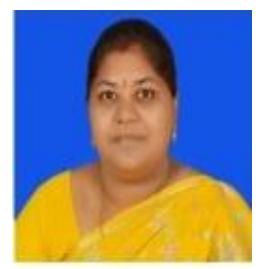

Radha Madhavi, was born in Tenali, India, in 1979. She received the M.Sc degree in Applied Mathematics from the Sri Padmavathi Mahila University, Tirupathi, India, in 2000, and the M.Phil. and Ph.D. degrees in Applied Mathematics from the Andhra University, Andhra Pradesh, India, in 2002 and 2013, respectively. In 2002, she joined the Department of mathematics, M.V.G.R.College of Engineering as a Assistant professor, and in 2007 became a Sr. Assistant professor. In 2009 She Joined as Sr. Assistant Professor in Velammal Institute of Technology, Chennai. In 2015 She joned in AVIT, Vinayaka missons University, Chennai as Sr. Associate professor to till date. Her Current research interests are Applications of Group theory and Graph Theory. 